\title{
Visitantes florales de Sanchezia oblonga (Acanthaceae) en un bosque montano de Carpish, Perú
}

\author{
Floral visitors of Sanchezia oblonga (Acanthaceae) \\ from a montane forest of Carpish, Peru
}

Rosa Villanueva-Espinoza ${ }^{1,2,}$ y Luis Pillaca-Huacre ${ }^{3}$

Recibido: 01 julio 2020 | Aceptado: 31 julio 2020 | Publicado en línea: 01 agosto 2020 Citación: Villanueva-Espinoza, R; Pillaca-Huacre, L. 2020. Visitantes florales de Sanchezia oblonga (Acanthaceae) en un bosque montano de Carpish, Perú. Revista Forestal del Perú 35(1): 73-79. DOI: http://dx.doi.org/10.21704/rfp.v35i1.1477

\begin{abstract}
Resumen
Las especies de Sanchezia cumplen un importante rol ecológico al proveer de alimento a otras especies, pero aún se conoce poco sobre esta interacción. Se presenta un estudio sobre la frecuencia de los individuos de los visitantes florales en Sanchezia oblonga durante la época húmeda, en un bosque montano en Carpish (Huánuco, Perú). Las observaciones tuvieron dos intervalos de 20 minutos en una inflorescencia de cinco individuos de S. oblonga. Se registraron cuatro grupos de visitantes, Diptera, Apidae, Formicidae y Trochilidae. Las frecuencias de visitas fueron de 2 a 6 individuos por inflorescencia, con varianza de $\pm 1.4 \mathrm{a} \pm 2.8$ individuos. Además, el promedio de la frecuencia de visitas de hormigas fue de $1.1 \pm 0.4$ individuos, similar al total de las otras familias de insectos juntas, sin embargo, se observó que las hormigas participaban como agentes "destructores" de los órganos florales.
\end{abstract}

Palabras clave: agente destructor, frecuencia de visitas, Huánuco, interacción

\footnotetext{
${ }^{1}$ Herbario Forestal MOL, Facultad de Ciencias Forestales, Universidad Nacional Agraria La Molina (UNALM), Av. La Molina s/n, La Molina, Lima, Perú.

${ }^{2}$ División de Ecología Vegetal - CORBIDI, Calle Santa Rita 105 Of. 2, Urb. Huertos de San Antonio Monterrico, Surco, Lima, Perú.

${ }^{3}$ Departamento de Ecología, Museo de Historia Natural, Universidad Nacional Mayor de San Marcos, Av. Arenales 1256, Jesús María, Lima, Perú.

* Autor de Correspondencia: rosvillanuevae@gmail.com
} 


\begin{abstract}
Sanchezia species plays an important ecological role by providing food to other species, but this interaction is still poorly understood. We present a study of the frequency of the individuals of the floral visitors in Sanchezia oblonga during the wet season, from a montane forest in Carpish (Huánuco, Peru). Observations had 20-minute two intervals in an inflorescence of five S. oblonga individuals. We recorded 4 groups of visitors, Diptera, Apidae, Formicidae y Trochilidae. Visitation frequencies ranged from 2 to 6 individuals per inflorescence, with a variance from \pm 1.4 to \pm 2.8 individuals. Additionally, the frequency mean of ant visits were $1.1 \pm 0.4$ individuals, similar to the total of the other insect families together, however, ants were observed to partake as destructive agents of floral organs.
\end{abstract}

Wey words: destructive agent, visits frequency, Huánuco, interaction

\section{Introducción}

El género Sanchezia Ruiz \& Pav. con alrededor de 56 especies herbáceas o arbustivas, es uno de los géneros más numerosos de la familia Acanthaceae Juss. (Tripp y Koenemann 2015). Sanchezia está restringida para el Neotrópico con centro de diversidad en Ecuador y Perú, registrándose 48 especies para la flora peruana (Villanueva-Espinoza y Condo 2019). En el Perú, el departamento de Huánuco, principalmente Tingo María y la Cordillera de Carpish, son considerados como zonas donde las especies de Acanthaceae alcanzan su punto máximo de endemismos en los Andes (Beck et al. 2007).

La mayoría de las especies de Sanchezia presentan una inflorescencia espiciforme con numerosas cabezuelas de monocasios permitiendo a las plantas abrir unas pocas flores en cada inflorescencia de forma secuencial durante un largo periodo de tiempo y, por lo tanto, proveer de un constante suministro de néctar (Schmidt-Lebuhn et al. 2005). Asimismo, las flores de las especies de Sanchezia presentan una corola grande y colorida con forma tubular. La presencia de estos caracteres sugeriría un síndrome de ornitofilia, principalmente por colibríes (Schmidt-Lebuhn et al. 2007). Esta interacción ha sido estudiada en pocas especies como Sanchezia oblonga Ruiz \& Pav., Sanchezia parvibracteata Sprague \& Hutch., Sanchezia putumayensis Leonard y Sanchezia skutchii Leonard \& L.B. Sm. (Schmidt-Lebuhn et al. 2007; Rodríguez-Flores et al. 2012; Arruda et al. 2015; Barney 2019).
Sanchezia oblonga está distribuida en Perú, Brasil, Colombia, Ecuador y el norte de Bolivia siendo ampliamente cultivada en América del Norte, África y Asia (Leonard 1951; Wasshausen y Wood 2004; Wasshausen 2013). En el Perú, se pueden encontrar poblaciones silvestres, cultivadas y naturalizadas de S. oblo$n g a$, por lo que, es una especie ampliamente distribuida y presenta un amplio rango altitudinal entre los 0-2500 msnm. Asimismo, esta especie destaca por su producción de flores durante todo el año. Sin embargo, a pesar de la importancia ecológica de S. oblonga, no existe información sobre sus interacciones en el territorio peruano, siendo necesaria esta información para conocer su historia natural y estado de vulnerabilidad. El presente estudio evalúa la frecuencia y abundancia de visitantes florales en Sanchezia oblonga como parte de su sistema de polinización en el Corredor Carpish-Yanachaga.

\section{Materiales y Métodos}

Área de estudio: Se ubicó en los Bosques montanos de Carpish, cerca de la catarata "San Miguel" y zonas de cultivos, correspondiente al distrito de Chinchao, provincia de Huánuco, departamento de Huánuco, Perú (9²9'42.91" S, 7554’39.46” O) a 1027 msnm (Figura 1).

Visitantes florales: El muestreo se realizó en enero del 2020 durante la época de lluvia, en el atardecer entre 2:00 p.m. y 4:00 p.m. Para reducir el sesgo en relación con las variables altitudinales, climáticas y por presencia de 
diferentes especies de flora aledaña, se evaluó en una misma zona. Todos los individuos de $S$. oblonga estaban presentes en un bosque secundario ribereño con presencia de agricultura aledaña. Se escogieron cinco individuos distanciados 5 metros uno del otro y cada individuo fue denominado (A, B, C, D y E). En cada individuo de $S$. oblonga se evaluó la frecuencia de los individuos de los visitantes florales en una inflorescencia durante dos intervalos de 20 minutos. Los registros de los visitantes fueron importantes para determinar el promedio de la abundancia de visitas por inflorescencia. Adicionalmente, se tomaron fotografías de algunos visitantes florales para su posterior confirmación en gabinete (Figura 2).

\section{Resultados}

El promedio de las frecuencias de los individuos de los visitantes florales por intervalo de muestreo en las inflorescencias de Sanchezia oblonga varió de $2 \pm 1.4$ a $6 \pm 2.8$ individuos. De las cinco inflorescencias evaluadas, sólo A, B y D presentaron el promedio de frecuencias de individuos de visitas con mayor variación en comparación con $\mathrm{C}$ y $\mathrm{E}$ que fueron visitados por la misma cantidad de individuos en los dos intervalos de muestreo, con 4 y 3 individuos en promedio respectivamente (Figura 3). Se registraron cuatro grupos taxonómicos de visitantes: Apidae, Diptera, Formicidae y Trochilidae. De ellas, Formicidae presentó el mayor promedio de visitas por inflorescencia (con $1.1 \pm 0.4$ individuos), seguido por Apidae (con $1 \pm 0.2$ individuos) (Figura 4). Durante el estudio se observó que las hormigas (Formicidae) destrozaban las corolas y los estambres, considerándose como "agentes destructores" para las flores (Figura 5). Además, se registró que el promedio de las frecuencias de visitas de las hormigas (Formicidae) por inflorescencia fue de $1.1 \pm 0.4$ individuos, valor similar al
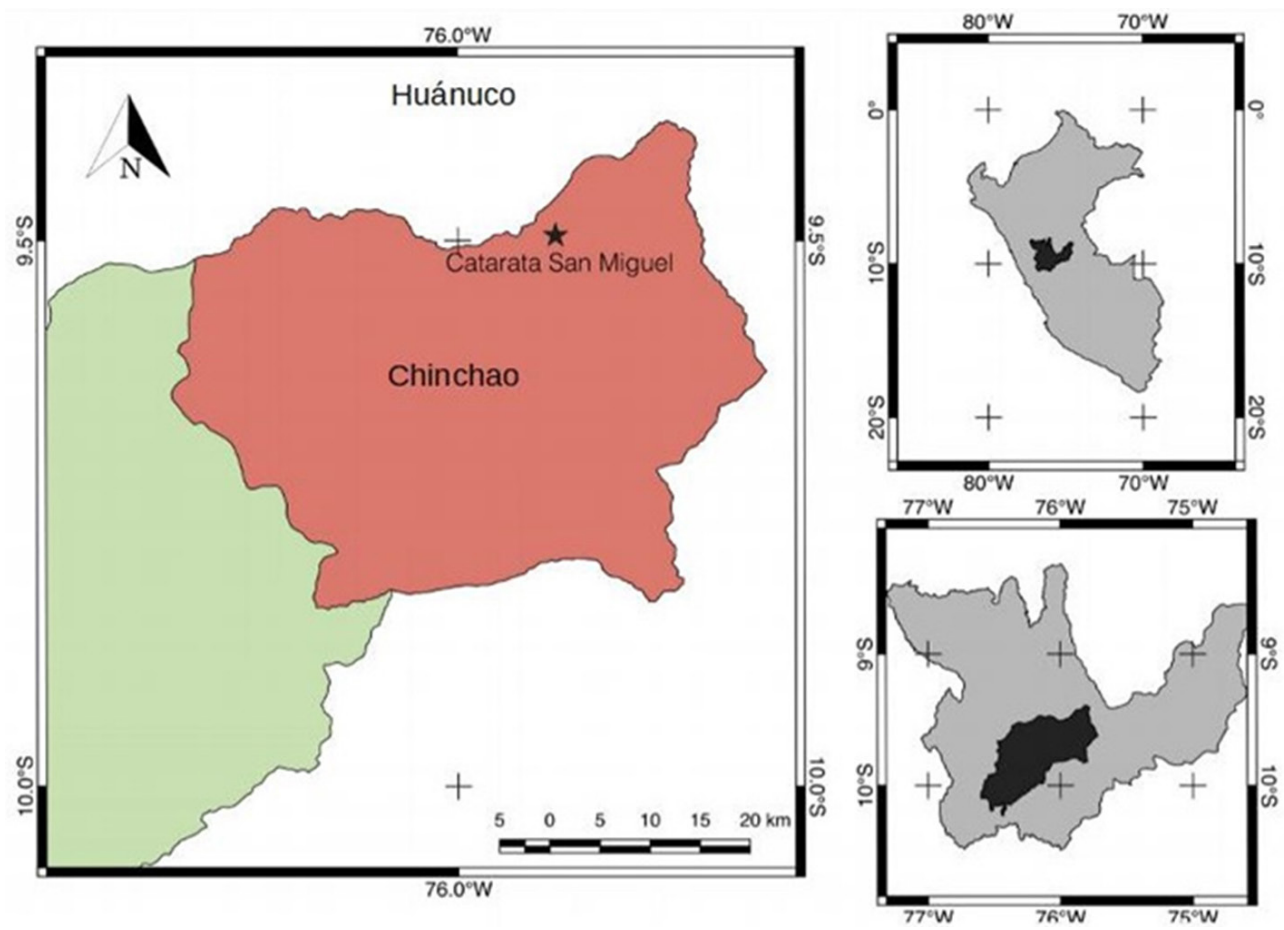

Figura 1. Ubicación de la zona de estudio. 


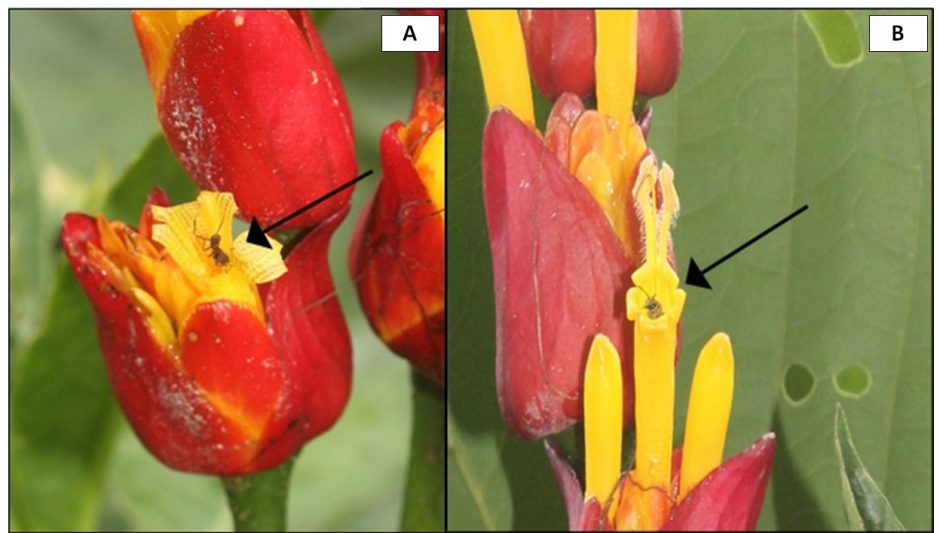

Figura 2. Visitantes florales en Sanchezia oblonga: A) Formicidae, B) Apidae.

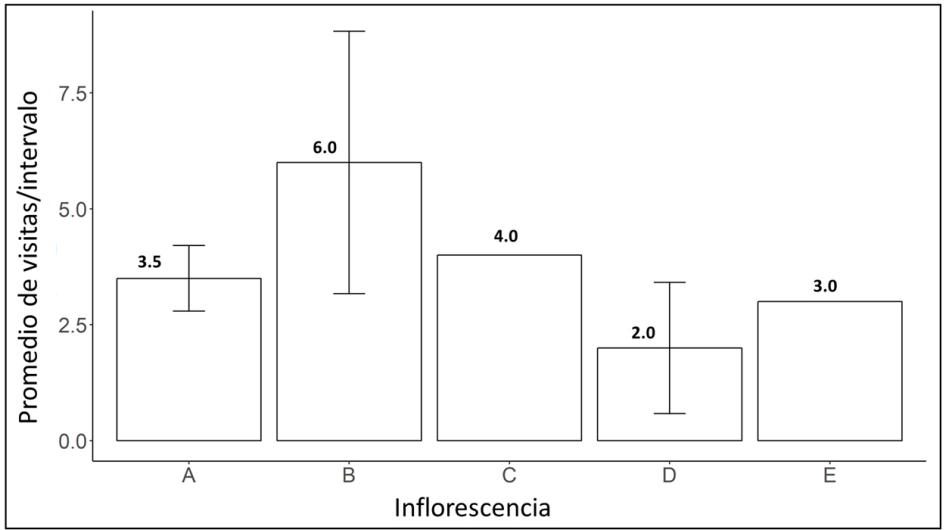

Figura 3. Visitantes florales por inflorescencia.

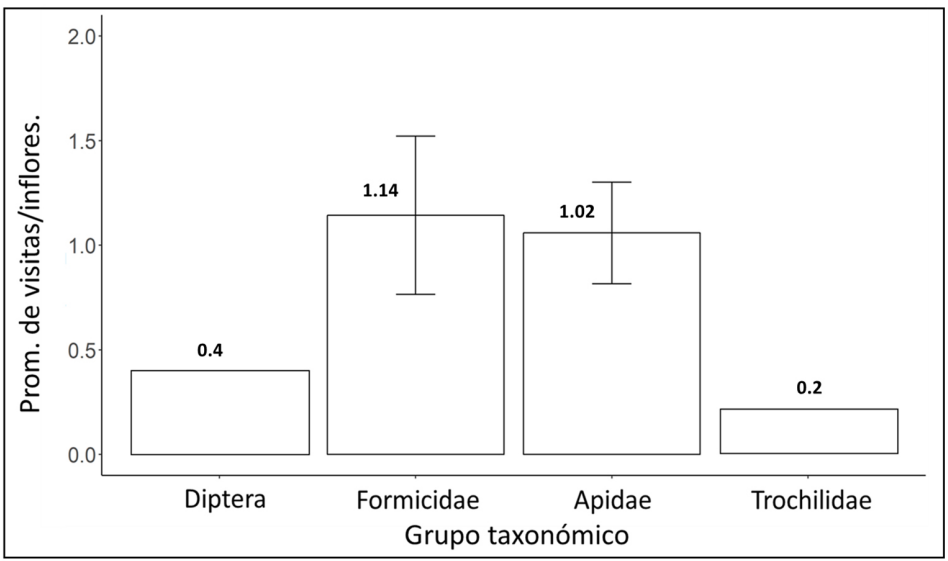

Figura 4. Frecuencia de individuos por familia de visitantes florales. 
promedio total de los otros insectos (Apidae y Diptera), con $1.4 \pm 0.2$ individuos por inflorescencia (Figura 6).

\section{Discusión}

La actividad de los visitantes florales generalmente disminuye durante épocas de lluvias y, por ende, el número de visitas a las flores son menores (Totland 1994), esto concuerda con la poca frecuencia de visitantes florales en los individuos evaluados de Sanchezia oblonga pese a la gran oferta de flores. Los representantes de Sanchezia poseen como polinizadores por excelencia a los colibríes (Trochilidae) (Schmidt-Lebuhn et al. 2007). Sin embargo, sólo se registró un individuo de una especie de colibrí en Sanchezia oblonga, en contraste con otros estudios que han registrado más de una especie (Schmidt-Lebuhn et al. 2007; Arruda et al. 2015; Silva y Consolaro 2015).

La presencia de algunos visitantes florales no indica necesariamente que sean los polinizadores de Sanchezia oblonga, debido a que estos visitantes (Apidae, Formicidae y Diptera) sólo se observaron de manera superficial en las flores y no ingresando a lo largo de la corola (McMullen 1995; Arruda et al. 2015). La pre- sencia de varios grupos taxonómicos por parte de los visitantes florales nos podría indicar en este tipo de eventos $S$. oblonga es una especie con interacción generalista, es decir, que recibe la presencia de vistas de diferentes grupos taxonómicos, como ocurre de manera reiterada en la interacción planta-visitante (Memmott 1999; Gómez 2002).

El promedio de visitas de hormigas (Formicidae) en Sanchezia oblonga fue similar al promedio total de los otros insectos visitantes, esto se puede deber a que las interacciones entre plantas-hormigas son muy frecuentes y participan de dos maneras: (1) de forma positiva, al interrumpir la herbivoría de otros "agentes destructores" que afectan hojas o flores, o (2) de manera negativa, al participar en la interrupción de la polinización, ya sea como causantes de herbivoría o como destuctores de órganos reproductivos de las flores (Styrsky y Eubanks 2007; Ballantyne 2011). Este último caso fue observado en $S$. oblonga cuyas flores (estambres y corola) fueron destruidas por las hormigas, formando parte del desincentivo en el momento de visitar las flores.

La heterogeneidad de los visitantes florales se debe a la secuencia de factores ecológicos, ambientales y genéticos, que dan como resul-

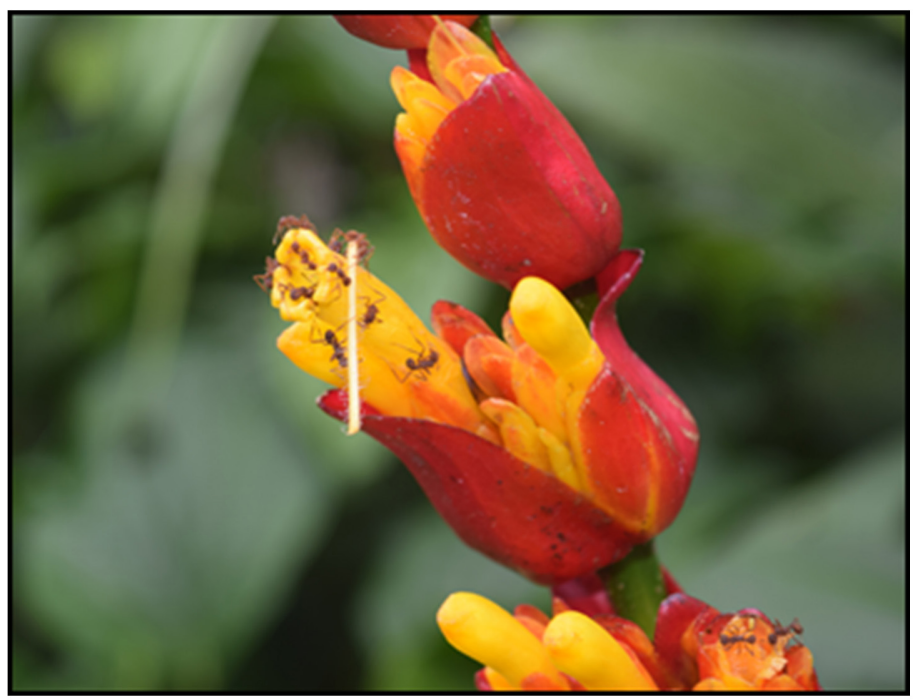

Figura 5. Presencia de "agentes destructores" en flores de Sanchezia oblonga. 


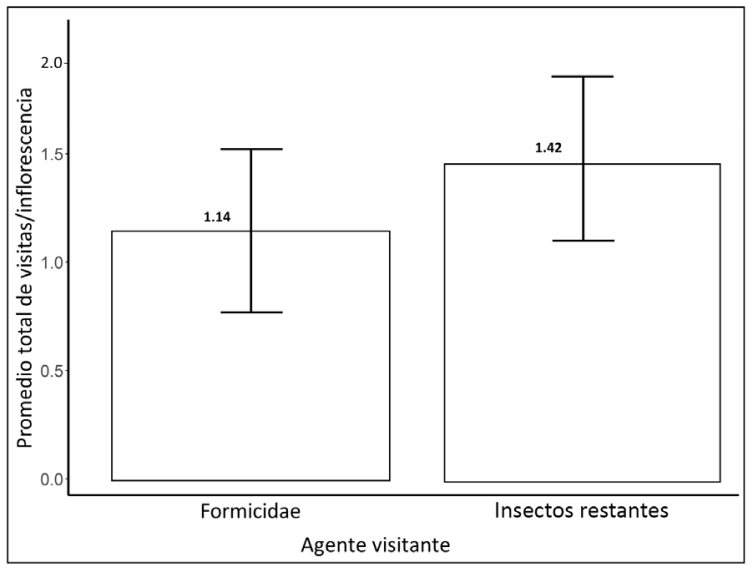

Figura 6. Promedio de visitas por tipo de "agente" visitante.

tado la interacción que tienen con las plantas. Esta relación principalmente se da por oportunismo (robo de néctar, lugar de reposo, depredación) o por ser agentes polinizadores como tal. Asimismo, esta interacción también depende de las temporadas donde se registran la mayor producción de flores de esta especie, siendo menor el forrajeo en épocas con lluvias constantes, las cuales limitan el movimiento de los visitantes florales. Tener una base de datos general sobre el sistema de polinización de plantas, permitiría conocer quiénes y cuántos son los visitantes florales en cualquier estudio de interacción planta-polinizador, considerando la frecuencia, diversidad y tipo de hábitat como base de correlación. Sin embargo, se requiere continuar con estas investigaciones para comprender mejor este tipo de interacción.

\section{Agradecimientos}

A APECO (Asociación Peruana para la Conservación de la Naturaleza) por el financiamiento brindado, mediante el premio para la Conservación "Carlos Ponce del Prado", proyecto "Promoviendo la investigación en las ACB priorizadas en Perú del Hotspot Andes Tropicales en apoyo a la conservación en el Perú" (CEPF-PROFONANPE-APECO, 2019). A Walter Espíndola por la elaboración del mapa de distribución, a Akira Wong por sus consejos y apoyo en el desarrollo del manuscrito y, a Robin Fernandez por el apoyo durante la fase de campo. Por último, a Igor Azevedo por compartir informaciónn sobre el género Sanchezia.

\section{Bibliografía}

Arruda, R.; Gomes-Pimentel, R.; Araújo, A.; Braz, D. 2015. Acanthaceae do Jardim Botânico da UFRRJ e seus visitantes florais. Anais. $3^{\text {a }}$ Reunião Anual de Iniciação Científica (2015, Seropédica, Brasil). Seropedica, Brasil, Universidade Federal Rural do Rio de Janeiro.

Ballantyne, G. 2011. Ants as flower visitors: floral ant-repellence and the impact of ant scentmarks on pollinator behavior. Tesis PhD. St Andrews, Escocia, University of St. Andrews. $170 \mathrm{p}$.

Barney, S. 2019. Management effects on hummingbird abundance and ecosystem services in a coffee landscape. Tesis Mag. Ann Arbor, Estados Unidos de America, University of Michigan. $30 \mathrm{p}$.

Beck, S.; Hernandez, P.; Jorgensen, P.; Paniagua, L.; Timaná, M.; Young, B. 2007. Plantas vasculares. In Young, B. (Ed.). Distribución de las especies endémicas en la vertiente oriental de los Andes del Perú y Bolivia. Virginia, Es- 
tados Unidos de América, Arlington: NatureServe. p. 18-34.

Gómez, J. 2002. Generalizations in the interactions between plants and pollinators. Revista Chilena de Historia Natural 75:105-116.

Leonard, E. 1951. The Acanthaceae of Colombia. Contribution from the United States $\mathrm{Na}$ tional Herbarium 31(1-3):1-781.

McMullen, C. 1995. Disponibilidad de polinizadores: una posible explicación para la variación floral entre islas de Justicia galapagana (Acanthaceae). Noticias de Galapagos 54-55:8-13.

Memmott, J. 1999. The structure of a plant-pollinator food web. Ecology Letters 2:276-280.

Rodríguez-Flores, C.; Stiles, F.; Arizmendi, M. 2012. Pollination network of a hermit hummingbird community (Thochilidae, Phaetornitidae) and their nectar resources in the Colombian Amazon. Ornitologia Neotropical 23:85-100.

Schmidt-Lebuhn, A.; Kessler, M.; Müller, J. 2005. Evolution of Suessenguthia (Acanthaceae) inferred from morphology, AFLP data and ITS rDNA sequences. Organisms Diversity \& Evolution 5:1-13.

Schmidt-Lebuhn, A.; Schwerdtfeger, M., Kessler, M.; Lohaus G. 2007. Phylogenetic constraints vs. ecology in the nectar composition of Acanthaceae. Flora 202:62-69.

Silva, R.; Consolaro, H. 2015. Polinização e sistema reprodutivo de Acanthaceae Juss. no Brasil: uma revisão. Bioscience Journal, Uberlândia 31(3):890-907.

Styrsky, J.; Eubanks, M. 2007. Ecological consequences of interactions between ants and honeydew-producing insects. Proceedings of the Royal Society London B 274:151-164.

Totland, O. 1994. Intraseasonal variation in pollination intensity and seed set in an alpine population of Ranunculus acris in southwestern Norway. Ecography 17:159-165.

Tripp, E.; Koenemann, D. 2015. Nomenclatural Synopsis of Sanchezia (Acanthaceae), Fifty
Years Since Last Treated. Novon: A Journal for Botanical Nomenclature 24(2):213-221.

Villanueva-Espinoza, R.; Condo, F. 2019. Sinopsis de la Familia Acanthaceae en el Perú. Revista Forestal del Perú 34(1):21-40.

Wasshausen, D.; Wood, J. 2004. Acanthaceae of Bolivia. Contribution from the United States National Herbarium 49:1-152.

Wasshausen, D. 2013. Acanthaceae. In Persson, C.; Stahl, B. (Eds.). Flora of Ecuador. Goteborg, Suecia: University of Gothenburg. p. 1-328. 